\title{
DIETARY SALT INTAKE: HISTORY, ASSESSMENT, AND BENEFIT IN HYPERTENSIVE TREATMENT
}

\author{
SUNITHA ESTHER RAJ, TAN LEE MEI, ADYANI MD REDZUAN*
}

Faculty of Pharmacy, Universiti Kebangsaan Malaysia, Jalan Raja Muda Abdul Aziz, 50300 Kuala Lumpur, Malaysia. Email: adyani@ukm.edu.my

Received: 15 June 2016, Revised and Accepted: 27 June 2016

\section{ABSTRACT}

Multiple lines of investigation including genetic, epidemiological, and interventional studies have demonstrated consistently a positive relationship between salt intake, blood pressure (BP) increment, and cardiovascular consequences. In addition, it has been documented that excessive salt intake can be attributed to various health complications such as asthma, osteoporosis, obesity, and gastric cancer. On the contrary, a reduction in salt intake has been shown to reduce BP and improve health outcomes, although the evidence is not completely unequivocal. Despite this discrepancy, a lowsodium diet is widely being recommended to all hypertensive patients in particular, as evidence against its efficacy in conjunction with optimum hypertensive treatment is well established. Determination of salt intake among hypertensive patients is important since dietary salt restriction had been proven to improve BP control in conjunction with optimum pharmacological management. Various methods have been used to estimate sodium intake includes 24-hrs urinary sodium, overnight urinary sodium, spot urinary sodium/creatinine ratio, and dietary survey methods. Reducing population salt intake has been proven to be beneficial, preventing millions of deaths from cardiovascular disease and stroke, and reducing the burden on health services. Many individual countries around the globe have already taken action against reducing population salt intake. These strategies were either led by government, nongovernment organizations, or industry.

Keywords: Dietary sodium, Salt, Hypertension, Sodium measurement, Cost-effectiveness.

(C) 2016 The Authors. Published by Innovare Academic Sciences Pvt Ltd. This is an open access article under the CC BY license (http://creativecommons. org/licenses/by/4. 0/) DOI: http://dx.doi.org/10.22159/ajpcr.2016.v9s2.13483

\section{INTRODUCTION}

Sodium is an essential element that is required in small amounts for the normal functioning of the body. Sodium ions together with chloride ions make up an inorganic compound known as salt. Salt is useful to human life because it improves taste and preserve food, especially during winter. However, the majority of people worldwide consume far more sodium, mostly from salt, than is required physiologically [1] In the United States for example, the average sodium consumption of adults was $3400 \mathrm{mg} /$ day, well above the current federal guideline of $<2300$ mg daily [2].

Excess dietary sodium has been linked to elevations in blood pressure (BP). Recent pre-clinical and clinical data supported that excess dietary sodium intake has also been associated with unwanted effects on calcium and bone metabolism, raised of risk in stomach cancer, aggravating asthma and affecting target organs such as heart, kidneys, and brain [3].

The fluid retention that occurs when excessive amounts of salt are consumed can induce or aggravate symptoms of congestive cardiac failure.

Excessive salt intake has also been implicated as a causative or aggravating factor in a number of other non-cardiac conditions such as Meniere's syndrome, kidney failure, hepatic failure, kidney stones, and pre-menstrual syndrome [4]. The American Heart Association also supports that excessive levels of sodium may put an individual at risk of developing stroke, osteoporosis, stomach cancer, kidney disease, enlarged heart muscle, and headaches [5].

According to Brown et al. [6] lowering the salt intake of individuals can shift the population distribution of BP toward more optimal levels, thus preventing millions of deaths from cardiovascular disease and stroke, and reducing the burden on health services. Sodium reduction is also cost-effective and beneficial has been proven by the national salt reduction strategies implemented in certain countries worldwide.

\section{EARLIEST HISTORY OF SALT AND HYPERTENSION}

Historically, the first ever record that relates dietary salt intake and BP were in $1700 \mathrm{BC}$ by a Chinese physician by the name of Huang TiNei Ching Su Wein, who said that "therefore if large amounts of salt are taken, the pulse will stiffen and harden" [7]. This idea paved numerous investigations on the association of high salt intake to the pathogenesis of hypertension. However, the results are mostly circumstantial [8]. In 1904, Ambard and Beaujard reviewed the salt hypothesis and decided that salt is necessary in the development of essential hypertension [9]. The papers, written in French and published in French scientific journal, were highly criticized as unconvincing. Soon in 1920s, 2 papers focusing on the effects of salt restriction as an anti-hypertension remedy were published [10]. Since then, the association of salt intake and health has slowly gain recognition. Still, it was not impactful enough to influence the medical opinion of those years.

Evidence-based research in the early $20^{\text {th }}$ century showed the absence of hypertension in patients who consumed $<3 \mathrm{~g}$ of salt daily. The BP reading was not raised as in comparison with those who consumed more than $20 \mathrm{~g}$ of salt per day, had BP readings above 140/90 $\mathrm{mmHg}$. In addition to that Walter Kempner was the first scientist who reported a consistent effect on BP via a dietary manipulation. He later termed the diet as Kempner rice diet which has a characteristically low content of salt [10]. His work was further confirmed with another successful experimentation done by Corcoran who concluded that the rice diet is, in fact, a practical low sodium diet, the hypothesis effect of which is dependent on sodium restriction [11].

During the mid-1900s, the Japanese discovered that the occurrence of cerebral hemorrhage has a regional distribution that directly correlated to the dietary intake of salt and the BP. They found that salt intake was also on the rise as high as $27 \mathrm{~g} /$ day. Out of this population, about $70 \%$ of the population had raised BP readings of more than $150 / 90 \mathrm{~mm} / \mathrm{Hg}$ [7]. This was compared to the population in the south of Japan where they consumed about $14 \mathrm{~g}$ of salt per day and showed to have a lower incidence of cerebral hemorrhage and only $10 \%$ had raised BP [7]. 
Work of Louis Dahl in 1960 marked the passage from historical to modern era in hypertension research. Dahl drew a famous linear graph that showed the positive relationship between the prevalence of hypertension and salt intake. He suggested that people needed physiologically about $1 \mathrm{~g}$ of salt each day but were consuming about $10 \mathrm{~g}$ of salt a day during his time [12]. Dahl also mentioned that an individual's dietary salt intake was difficult to measure as it was highly variable. Dahl recommended that the $24 \mathrm{hrs}$ urine excretion was the best method available. Finally, Dahl also postulated that hypertension would be an area of great concern if people were consuming more than 4-5 g of salt per day [12].

\section{WORLDWIDE POPULATION STUDY ON SALT REDUCTION AND BENEFIT IN HYPERTENSION}

Research on the role of salt in the pathogenesis of hypertension was greatly intensified spanning anthropology, epidemiology, animal studies, studies of mechanisms, and clinical trials since 1960. The most referred to study is the INTERSALT study [13]. This study was an international observational study carried out on 10,079 subjects in 52 centers in 32 countries worldwide to investigate the salt-BP relationship. BP readings were measured for each subject together with sodium and potassium urine concentrations. In each center, the subject's BP reading was plotted with age and then later with salt concentration. They found that when the regression values were pooled, the results were highly significant. Sodium excretion correlated with the increase of BP with age. On the other hand, potassium is inversely associated with BP of individuals [14]. The authors concluded that a reduction in about 100 $\mathrm{mol} /$ day of salt will lead to a reduction of 1-6 $\mathrm{mmHg}$ of systolic pressure and about 0.03-2.5 mmHg in diastolic pressure.

In 1996, following an objection from the Salt Institute on INTERSALT's findings, the data were reassessed to compare quantitative estimates of the sodium-BP relations from ecological and individual analyses. On the reassessment on data, they found that effect of median sodium excretion higher by $100 \mathrm{mmol} /$ day over a 30-year period were of greater difference of 10-11 $\mathrm{mmHg}$ in systolic BP and $6 \mathrm{mmHg}$ in diastolic BP, hence encouraging the general population to reduce salt intake [15].

Another interventional study includes Trial of Hypertension Prevention (TOHP) and Dietary Approaches to Stop Hypertension (DASH) $[16,17]$ TOHP is a clinical interventional trial to test the short-term feasibility and efficacy of seven non-pharmacological interventions in person with high normal BP, aimed at preventing hypertension. They are three lifestyle changes (weight reduction, sodium reduction, and stress management) and four nutritional supplement groups (calcium, magnesium, potassium, and fish oil). Of these, weight reduction and sodium reduction were found to be the most effective strategy in reducing $\mathrm{BP}$ in normotensive person with reduction of $1.7-2.9 \mathrm{mmHg}$ systolic and 0.9-2.3 mmHg diastolic BP reading.

Conversely, DASH is a diet plan designed for people who aim for weight loss, have diabetes and even for hypertension. It is an eating plan to help maintain good health. In the landmark DASH-sodium trial which was a multi-centerd clinical trial conducted on 412 subjects with and without hypertension, three levels of sodium intake were administered to the subjects with either the DASH diet or a controlled diet [17]. The results showed that the lower intake of sodium produced better BP readings with the DASH diet as compared to the controlled diet. When the lowest and moderate levels of sodium were compared, it was found to have a $6.7 / 3.5 \mathrm{mmHg}$ difference in systolic versus diastolic reading pressure respectively. Finally, when the moderate and high levels of sodium were compared, the results showed a $4.6 / 2.1 \mathrm{mmHg}$ drop in systolic and diastolic BP readings. The authors concluded that most people consume about $10 \mathrm{~g}$ of salt per day which is equivalent to approximately $170 \mathrm{mmol}$ of sodium (Sacks et al. 2001). The dietary guide for Americans (2005) suggests $2300 \mathrm{mg}$ of sodium a day for the general population and about $1500 \mathrm{mg}$ of sodium for hypertensive patients [12].
A systematic meta-analysis on the effect of lower sodium intake reported that a reduction of sodium intake has a significant reduction in systolic $\mathrm{BP}$ by about $3.4 \mathrm{mmHg}$ and diastolic of $1.5 \mathrm{mmHg}$. When sodium intake was $<2 \mathrm{~g}$ /day, systolic BP decreased by $3.5 \mathrm{mmHg}$ while diastolic reading dropped by $1.8 \mathrm{mmHg}$. However in this study, a decrease in sodium intake was associated with an increase in the lipid profile, catecholamine levels, and renal impairment. The authors also reported that an increase in sodium intake leads to an increase in the incidence of stroke, risk of stroke mortality as well as risk of coronary heart disease [18].

Another meta-analysis of randomized trials on the modest salt reduction on BP states that there is very little effect in reducing salt intake over a short period of time. The authors analyzed 17 trials with a median urine sodium excretion of $78 \mathrm{mmol}$ in hypertensive patients and $74 \mathrm{mmol}$ in normotensive patients. The BP drop in the hypertensive patients was recorded at $4.96 / 2.73 \mathrm{mmHg}$ in the hypertensive group while in the normal group the BP reading drop was recorded as $2.03 / 0.97 \mathrm{mmHg}$. The authors concluded that reducing salt intake over a long-term period helps in the prevention of stroke and coronary heart disease in hypertensive patients [19].

In a more recent meta-analysis in 2013, he and colleagues included 34 randomized trials of 3230 participants, both with raised BP and normal BP. With a reduced sodium excretion of $75 \mathrm{mmol} / 24 \mathrm{hrs}$, the mean change in BP was $-4.8 \mathrm{mmHg}$ systolic and -2.06 for diastolic BP. After adjustment for age, ethic group and BP status, a $100 \mathrm{mmol}$ reduction in daily sodium resulted in a fall of systolic BP of $5.8 \mathrm{mmHg}$. The metaanalysis concluded that a modest reduction in salt intake reduces BP significantly both in hypertensive and normotensive people.

\section{VARIOUS METHODS FOR DETERMINATION OF DIETARY SALT INTAKE}

The study of salt and BP has utilized several methods in measuring the amount of salt a person consumes from the most reliable 24-hrs urine collection to an analysis from the salt frequency questionnaire. Some of the most commonly used methods are dietary recall method or also known as the $24 \mathrm{hrs}$ record, a single food frequency questionnaire (FFQ) done together with a $24 \mathrm{hrs}$ urine collection as well as multiple FFQs. Most dieticians have agreed that it is difficult to measure the amount of salt a person consumes as one is unable to determine the amount of salt remaining on the serving plate. This congruent was also noted in Dahl's 1960 article where he remarks that an individual's dietary salt intake is highly variable and difficult to measure [12]

Even the 24 hrs urine collection, which is considered as the "gold standard" to measure sodium intake is argumentative as it only captures $85-90 \%$ of ingested sodium [20]. Therefore, the most appropriate method to estimate the amount of salt consumed in a day would be to use both the dietary salt questionnaire together with the $24 \mathrm{hrs}$ urine collection. This would help estimate the amount salt in each food item consumed over the last 2-3 months and the $24 \mathrm{hrs}$ urine collection will help quantify the amount of sodium excreted (Table 1).

Measurement of 24 hrs urine dietary sodium is also fraught with a reduced response rate due to a number of factors such as high cost

Table 1: Limitation of 24-hrs urine collection [21]

\begin{tabular}{l}
\hline Limitation of 24-hrs urine collection \\
1. Participant burden is high and therefore rates of incomplete \\
collection/attrition may be high \\
2. The collection must be complete, with no more than a few drops \\
lost, otherwise the excretion estimate will be biased \\
3. There is no absolute check on completeness, though good survey \\
technique may help to reduce levels of incompleteness \\
4. The collection must be accurately timed to avoid over-as well \\
as under-collection and so that minor deviations from a 24-hrs \\
collection period can be corrected
\end{tabular}


and participant burden, time-consuming and inconvenient to the individual performing the collections and frequent incident of undercollection [22]. A simpler and alternative method would be to use a spot urine sample. Japanese investigators including Kawasaki and Tanaka have demonstrated that it is possible to estimate 24-hrs urinary sodium from single spot urine. Using second-morning void urine, Kawasaki successfully achieve correlation of $\mathrm{R}=0.85$ between second-morning void to 24-hrs urine collection [23]. Tanaka reported a correlation of 0.65 between both methods and concluded that while spot urine is not suitable to estimate individual sodium excretion, it can be used to measure population intake of sodium [24]. Subsequently, spot urine sample is seen as a great replacement of the highly troublesome 24-hrs urine method. In 2013, inter salt attempted to estimate 24-hrs urine sodium excretion from casual urinary sodium concentration in Western population with 5693 participants. Correlation of 0.50 was observed which lead to a conclusion; casual urine sample may be useful, low burden, low-cost alternative to 24-hrs urine collection for estimation of population sodium intake. However, ongoing calibration with the study specific 24-hrs urinary collection is recommended to increase validity [21].

FFQ are created to assess habitual diet of patients by asking the frequency of which food items are consume over a period of time. This method of measuring sodium intake through estimation and calculation from questionnaire or food consumption data has been used a lot in studies that require monitoring of the diet habits [20]. It is the most common method used to assess dietary consumption of a population over a period of time. The performance of the FFQs has been compared to many more intensive, record based methods such as 24-hrs food recalls, food diaries or duplicates of food collection. The two well-known FFQ is the Willett questionnaire and the block questionnaire which have been used in many epidemiological studies [25].

FFQs can be used to identify food patterns inadequacy in relation to specific nutrients. Some common problems that have been identified with FFQs are over or under-estimation. Over-estimation is usually observed for foods that are often less eaten or "healthy food" such as vegetables [26]. In contrary, underestimation from discretionary salt is difficult to estimate and may not be captured by questionnaire. The measurement using questionnaires and surveys are often prone to errors and biases as participants usually relay on memory to estimate food intake. FFQs are also location and culture dependent due to the distinct variation of food based on culture value. As such, there is a need to constantly update the wide array of ever changing food databases used in FFQ [20].

Despite that, FFQs have been reported to be reliable and accurate in providing average diet consumption as well as are able to show the current trend of consumption. Due to this, the method is widely used in nutritional epidemiological studies and is a key tool in dietary studies. The advantage of using this method is the ease of administration and low patient burden. Furthermore, FFQs have a lower cost on the participants which allows the assessment to be conducted more than once if necessary [25]. A study done by Goulding et al. in 1998 showed that FFQs can be used to analyze the intake of specific nutrients such as calcium, potassium, and sodium. A review by Batcagan-Abueg et al. reported poor correlations between dietary survey and urine collection ranging from $r=0.09-0.30[20]$.

\section{COST-EFFECTIVENSS OF SALT REDUCTION PROGRAMME}

Recently, it has been estimated that increased BP accounts for US\$370 billion in health care costs worldwide and, in developed countries, accounts for approximately $10 \%$ of overall direct health costs. Indirect costs are estimated at US\$3.6 trillion worldwide [26]. In Canada, direct health care savings of $\$ 1.38$ billion per year would be achieved by lowering sodium of about $1800 \mathrm{mg} /$ day, and the indirect costs of savings total up would be $\$ 2.99$ billion/year [27].
Reducing the salt consumption globally was predicted to shift the population distribution of BP toward more healthy levels, thus millions of deaths from cardiovascular disease and stroke can be prevented while reducing the pressure on health services. Sodium reduction can lower down the global burden of cardiovascular disease, in a more costeffective and efficient way [6].

Besides, global estimates suggested that a $9.5 \%$ reduction in salt consumption could prevent 513,885 strokes and 480,358 myocardial infarctions over the lifetime of adults aged 40-85 years, increasing quality-adjusted life years (QALYs) by 2.1 million and saving $\$ 32.1$ billion in medical costs. A tax on sodium that decreases population sodium intake by $6 \%$ will increase QALYs by 1.3 million and saves $\$ 22.4$ billion over the same period [28].

A study published in the United States showed that a reduction of salt consumption by $3 \mathrm{~g}$ (1200 mg sodium)/day helped to save 194,000 and 392,000 QALYs, and save between $\$ 10$ billion and $\$ 24$ billion in health care costs every year. Even with a decline of $1 \mathrm{~g}$ salt ( $400 \mathrm{mg}$ sodium)/day would result in decreasing of 20,000-40,000 number of coronary heart disease cases, 11,000-23,000 number of stroke cases, $18,000-35,000$ myocardial infarction cases, and deaths from all causes by between 15,000 and 32,000 every year [29].

\section{CONCLUSION}

Excessive intake of dietary sodium may lead to various health complications with hypertension being the main concern. Pharmacotherapy coupled with lifestyle modification is strongly emphasized for hypertensive patients to optimized the BP control. In the recent year, much effort has been made on lifestyle intervention such as monitoring and reduction of dietary salt intake. Point-of-care testing for sodium assessment utilizing spot urine analysis or FFQ among hypertensive patient is highly recommended. Perhaps it is timely to view hypertension treatment as a whole inclusive of patients' lifestyle background and pharmacotherapy treatment instead of drug therapy alone.

\section{REFERENCES}

1. He FJ, Macgregor GA. Salt, blood pressure and cardiovascular disease. Curr Opin Cardiol 2007;22(4):298-305.

2. Institute of Medicine. Sodium Intake in Populations: Assessment of Evidence. Washington, DC: The National Academies Press; 2013.

3. Farquhar WB, Edwards DG, Jurkovitz CT, Weintraub WS. Dietary sodium and healthmore than just blood pressure. J Am Coll Cardiol 2015;65(10):1042-50.

4. National Heart Foundation of Australia. Salt and Hypertension, (Professional Paper); 2007. p. 555

5. Go AS, Mozaffarian D, Roger VL, Benjamin EJ, Berry JD, Borden WB, et al. Heart disease and stroke statistics--2013 update: A report from the American Heart Association. Circulation 2013;127(1):e6-245.

6. Brown IJ, Tzoulaki I, Candeias V, Elliott P. Salt intakes around the world: Implications for public health. Int J Epidemiol 2009;38(3):791-813.

7. Meneton P, Jeunemaitre X, de Wardener HE, MacGregor GA. Links between dietary salt intake, renal salt handling, blood pressure and cardiovascular disease. Am Physiol Soc 2005;85(2):679-715.

8. Luft FC, Grim CE, Fineberg N, Weinberger MC. Effects of volume expansion and contraction in normotensive whites, blacks, and subjects of different ages. Circulation 1979;59(4):643-50.

9. Amiri M, Kelishadi R. Can salt hypothesis explain the trends of mortality from stroke and stomach cancer in Western Europe? Int J Prey Med 2012;3(6):377-8

10. Cirillo M, Del Giudice L, Bilancio G, Franzese MD, De Santo NG. Low salt diet and treatment of hypertension: An old story. J Nephrol 2009;22 Suppl 14:136-8.

11. Corcoran AC, Taylor RD, Page IH. Controlled observations on the effect of low sodium dietotherapy in essential hypertension. Circulation 1951;3(1):1-16

12. Elliot $P$. Role of salt intake in the development of high blood pressure. Int J Epidemiol 2005;34:975-8.

13. Intersalt: An international study of electrolyte excertion and blood pressure. Results for 24 hour urinary sodium and potassium 
excretion. Intersalt Cooperative Research Group. $\mathrm{Br}$ Med J 1988;297(6644):319-28.

14. Haddy FJ, Vanhoutte PM, Feletou M. Role of potassium in regulating blood flow and blood pressure. Am J Physiol Regul Integr Comp Physiol 2006;290(3):R546-52

15. Elliott P, Stamler J, Nichols R, Dyer AR, Stamler R, Kesteloot H, et al. Intersalt revisited: Further analyses of 24 hour sodium excretion and blood pressure within and across populations. Intersalt Cooperative Research Group. BMJ 1996;312(7041):1249-53.

16. Cook NR, Cutler JA, Obarzanek E, Buring JE, Rexrode KM, Kumanyika SK, et al. Long term effects of dietary sodium reduction on cardiovascular disease outcomes: Observational follow-up of the trials of hypertension prevention (TOHP). BMJ 2007;334(7599):885-8.

17. Sacks FM, Svetkey LP, Vollmer WM, Appel LJ, Bray GA, Harsha D et al. Effects on blood pressure of reduced dietary sodium and the Dietary Approaches to Stop Hypertension (DASH) diet. DASH-Sodium Collaborative Research Group. N Engl J Med 2001;344(1):3-10

18. Aburto NJ, Ziolkovska A, Hooper L, Elliott P, Cappuccio FP, Meerpohl JJ. Effect of lower sodium intake on health: Systematic review and meta-analyses. BMJ 2013;346:f1326.

19. He FJ, MacGregor GA. Effect of modest salt reduction on blood pressure: A meta-analysis of randomized trials. Implications for public health. J Hum Hypertens 2002;16(11):761-70.

20. Batcagan-Abueg AP, Lee JJ, Chan P, Rebello SA, Amarra MS. Salt intakes and salt reduction initiatives in Southeast Asia: A review. Asia Pac J Clin Nutr 2013;22(4):490-504.

21. Brown IJ, Dyer AR, Chan Q, Cogswell ME, Ueshima H, Stamler J, et al. Estimating 24-hour urinary sodium excretion from casual urinary sodium concentrations in Western populations: The INTERSALT study. Am J Epidemiol 2013;177:1180-92.

22. Charlton KE, Steyn K, Levitt NS, Jonathan D, Zulu JV, Nel JH Development and validation of a short questionnaire to assess sodium intake. Public Health Nutr 2008;11(1):83-94.

23. Kawamura M, Ohmoto A, Hashimoto T, Yagami F, Owada M Sugawara T. Second morning urine method is superior to the casual urine method for estimating daily salt intake in patients with hypertension. Hypertens Res 2012;35:611-6.

24. Tanaka T, Okamura T, Miura K, Kadowaki T, Ueshima H, Nakagawa H, et al. A simple method to estimate populational 24-h urinary sodium and potassium excretion using a casual urine specimen. J Hum Hypertens 2002;16:97-103.

25. Willett W. Commentary: Dietary diaries versus food frequency questionnaires-a case of undigestible data. Int $\mathrm{J}$ Epidemio 2001;30(2):317-9.

26. Gaziano TA, Bitton A, Anand S, Weinstein MC; International Society of Hypertension. The global cost of nonoptimal blood pressure. J Hypertens 2009;27(7):1472-7.

27. Penz ED, Joffres MR, Campbell NR. Reducing dietary sodium and decreases in cardiovascular disease in Canada. Can J Cardiol 2008;24(6):497-501.

28. Smith-Spangler CM, Juusola JL, Enns EA, Owens DK, Garber AM Population strategies to decrease sodium intake and the burden of cardiovascular disease: A cost-effectiveness analysis. Ann Intern Med 2010;152(8):481-7, W170-483.

29. Bibbins-Domingo K, Chertow GM, Coxson PG, Moran A, Lightwood JM, Pletcher MJ, et al. Projected effect of dietary salt reductions on future cardiovascular disease. $\mathrm{N}$ Engl J Med 2010;362(7):590-9 